\title{
Collinear and Non-Collinear Configurations in Classical Geometrically Frustrated Ring-Shaped Systems
}

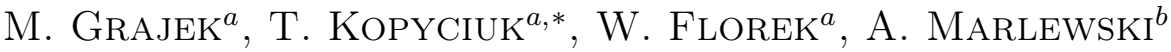 \\ ${ }^{a}$ Faculty of Physics, Adam Mickiewicz University in Poznań, Poland \\ ${ }^{b}$ Institute of Mathematics, Poznań University of Technology, Poland
}

\begin{abstract}
Geometrically frustrated quantum spin systems, with competing antiferromagnetic couplings, show the Kahn degenerate frustration for some specific values of Heisenberg Hamiltonian parameters. It has been recently shown for rings with a defect bond and centered rings. In the case of classical counterparts of these systems, degenerated configurations with the lowest energy are present for the energy function parameter greater than a certain threshold. In these domains such configurations are planar but non-collinear with continuous changes of the net magnetic moment with respect to the Hamiltonian parameter. Outside these domains there is unique collinear ground state configuration (neglecting choice of the net magnetic moment direction). However, these collinear configurations are the same in both non-frustrated and geometrically frustrated domains. Numerically exact calculations for quantum systems strongly confirm that determined properties of their classical counterparts realize the classical limit $s \rightarrow \infty$.
\end{abstract}

DOI: 10.12693/APhysPolA.133.417

PACS/topics: 75.10.Jm, 75.10.Hk, 75.50.Xx

\section{Introduction}

The concept of frustration has its origin in classical systems, especially in the frustrated Ising models and the spin glass theory [1-3]. Frustration is present when there exists at least one cycle of spins with non-zero exchange integrals between consecutive spins and an odd number of them are antiferromagnetic. This description is related to the system geometry, so the term geometric frustration is commonly used in this case. In such systems competing interactions are present and such approach can be easily adopted to many classical and semi-classical models, for example, the Potts model or the classical counterpart of the Heisenberg model $[4,5]$. The presence of competing interactions means that a system cannot simultaneously satisfy all the interactions that it undergoes. This leads to a more general concept of energetic frustration: a sum of the minimum energies for each individual bond (each term of an energy function) is less than the lowest energy of a system in question $[4,6]$.

In the case of finite quantum systems, 7 put stress on the the ground state degeneration introducing the notion of degenerate frustration $[7,8]$. This problem has been studied recently in the context of magnetic molecules [9-16] and it has been shown that in a certain domain of the Hamiltonian parameters, a geometrically frustrated spin system with competing interactions has the unique ground state $S$-multiplet, with the same total spin number $S$ as in the domain without frustration. This region was assigned to the third type of frustration, whereas the ground state multiplets with other values of $S$ represent the second type of frustration. The first

*corresponding author; e-mail: tomasz.kopyciuk@amu.edu.pl type corresponds to the degenerate frustration $[10,14]$. The third type of frustration is also observed in the Isinglike counterparts of models considered [17].

Frustration in classical spin systems can be also expressed in other words: non-frustrated systems have a collinear configuration with the lowest energy (LEC) $[6,18]$. Therefore, if the LEC is non-collinear then the system is frustrated. This condition does not exclude collinear LECs in frustrated systems. By analogy with quantum systems, it is referred to as the third type of frustration. In this paper two such systems are presented: antiferromagnetic odd-numbered rings and rings with an even number of spins uniformly coupled to an extra one. The first case is related to the $\mathrm{Cr}_{9}$ molecule [10], whereas the other is a model of $\mathrm{Fe}_{7}$ or $\mathrm{Gd}_{7}$ molecule $[19,20]$. In these cases there is a welldetermined domain with the collinear LEC despite the presence of frustration and this configuration is the same as in the absence of frustration. It can be shown that non-collinear LECs are planar in these models (cf. [18]), hence positions of all but one spins (vectors) $\boldsymbol{s}_{j}, j \neq p$, are determined by angles $\varphi_{j}$ they form with a fixed one $s_{p}$ and a set $\Phi=\left\{\varphi_{j}\right\}$, with a chosen $\varphi_{p}=0$, determines a configuration of spins. Hence, for a pair of spins $\boldsymbol{s}_{j}$ and $s_{j^{\prime}}$ the classical counterpart of the Heisenberg coupling yields $E_{j j^{\prime}}(\Phi)=J_{j j^{\prime}} \cos \left(\varphi_{j^{\prime}}-\varphi_{j}\right)$ (homogeneous systems are considered and it is assumed that $\left|s_{j}\right|=1$ ). Due to parity of the cosine function, non-collinear configurations are (at least) two-fold degenerated - configurations with opposite signs of all angles $\phi_{j}$ have the same energy, but different chirality. Therefore, non-collinear LECs should be assigned to the first type of frustration.

\section{Rings with a bond defect}

For a given set $\Phi$ of angles and non-zero couplings between the nearest neighbors only, the energy is written 
as $(n>1$ is an odd integer $)$

$$
E(\Phi)=\alpha \cos \xi+\sum_{j=1}^{n-1} \cos \psi_{j},
$$

where $\psi_{j}=\varphi_{j+1}-\varphi_{j}$ for $1 \leqslant j<n$ and $\xi=\varphi_{1}-\varphi_{n}$. Due to symmetry of the problem, it is convenient to put $\varphi_{p}=0$ for $p=(n+1) / 2$. When $\alpha<0$ the unique LEC is a collinear one with $\boldsymbol{s}_{j}=(-1)^{p+j} \boldsymbol{s}_{p}$, so $\psi_{j}=\pi$ and $\xi=0$. Therefore, this antiferromagnetic configuration $\Phi^{\mathrm{AF}}$ (Fig. 1a) has the energy

$$
E\left(\Phi^{\mathrm{AF}}\right)=\alpha-(n-1) ;
$$

the net magnetic moment equals $(-1)^{p-1} \boldsymbol{s}_{p}$.
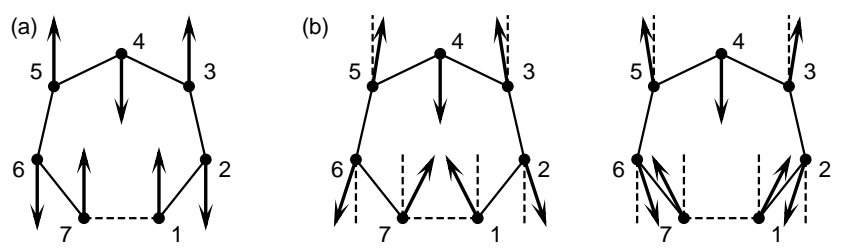

Fig. 1. The lowest energy configurations for a heptanuclear ring with a single bond defect $\left(J_{j j+1}=1\right.$ for $1 \leqslant j<7, J_{71}=\alpha$ ). (a) The unique configuration for $\alpha<\alpha_{\mathrm{c}}=1 / 6$ and (b) two configurations, with different chirality, for $\alpha>1 / 6$.

It can be shown that there are two non-collinear LECs with all angles $\psi_{j}=\psi$ and $\xi=(n-1)(\pi-\psi)$, where $0<|\xi|<\pi$ (Fig. 1b); a sign of $\xi$ determines chirality of the corresponding LEC, respectively. However, the relation

$$
\alpha=-\frac{\sin \psi}{\sin [(n-1) \psi]}=\frac{\sin [\xi /(n-1)]}{\sin \xi}
$$

between the energy function parameter $\alpha$ and the angle $\psi$ (or $\xi$ ) can be satisfied for $\alpha$ greater than a certain critical value $\alpha_{\mathrm{c}}$. This threshold can be easily found as $\lim _{\psi \rightarrow \pi} \alpha=1 /(n-1)$. Then the lowest system energy as a function of the parameter $\alpha$ is given by Eq. (2) for $\alpha \leqslant \alpha_{\mathrm{c}}$ and by Eq. (1) for $\alpha \geqslant \alpha_{\mathrm{c}}$, where the values of $\psi_{j}=\psi$ are determined from Eq. (3). The latter one reaches its maximum when $|\xi|=\pi / 2$, so at $\alpha_{\mathrm{c}}<\alpha_{\max }=\sin [\pi / 2(n-1)]<1$ (cf. [6] and Fig. 2). The net magnetization equals

$$
\boldsymbol{M}=\frac{\sin (n \psi / 2)}{\sin (\psi / 2)} \boldsymbol{s}_{p},
$$

what gives the non-magnetic LEC at $\alpha=1$ with $|\psi|=$ $|\xi|=(n-1) \pi / n$ (Fig. 3).

\section{Centered rings}

A spin vector $s_{0}$ is placed at the center of a regular polygon and the other $n$ vectors $\boldsymbol{s}_{j}, 1 \leqslant j \leqslant n$ (for an even number $n$ ), are placed at its vertices. Each configuration is determined by a set $\Phi=\left\{\varphi_{j}\right\}, 1 \leqslant j \leqslant n$, of angles they form with the vector $s_{0}$. The couplings are put to be $J_{j j+1}=1$ and $J_{0 j}=\alpha$ for $1 \leqslant j \leqslant n, n+1 \equiv 1$. Hence, the energy function is written as

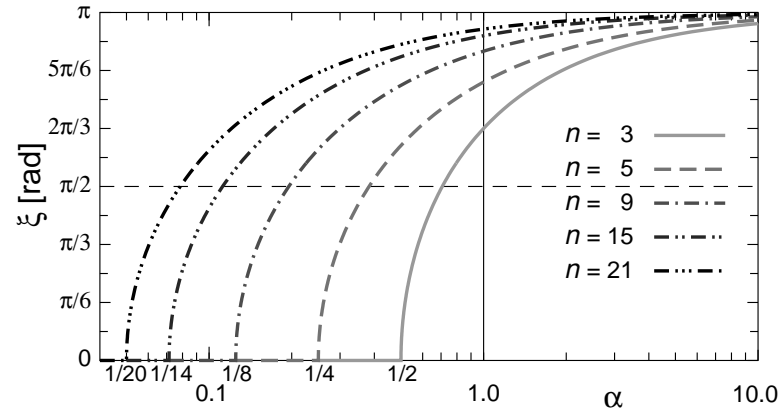

Fig. 2. The angle $\xi$ as a function of the parameter $\alpha$ for some rings with a single bond defect (log-linear scale).

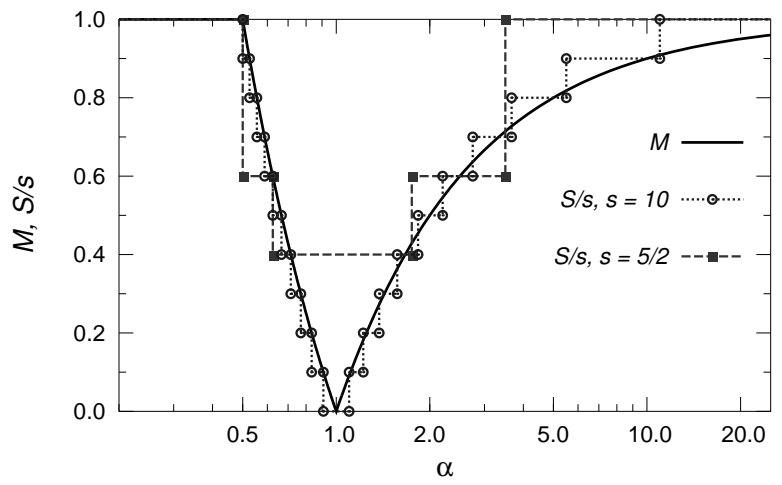

Fig. 3. An absolute value of the net magnetization $M=|\boldsymbol{M}|$ for an isosceles triangle within the classical Heisenberg model compared with a relative total spin number $S / s$ in its quantum counterpart for $s=10$ and $s=5 / 2$; see also $[14,16]$.

$$
E(\Phi)=\sum_{j=1}^{n} \cos \left(\varphi_{j+1}-\varphi_{j}\right)+\alpha \sum_{j=1}^{n} \cos \left(\varphi_{j}\right) .
$$

This system is geometrically frustrated for $\alpha \neq 0$; for $\alpha=0$ there are two isolated systems, so the ring is ordered antiferromagnetically, $\varphi_{j+1}-\varphi_{j}=\pi$, what gives $\varphi_{2 k-1}=\varphi, \varphi_{2 k}=\pi+\varphi, 1 \leqslant k \leqslant n / 2$, for any $0 \leqslant \varphi<2 \pi$. The non-frustrated counterpart of this system is realized for ferromagnetic couplings within the ring, $J_{j, j+1}=-1$. In this case collinear LECs are determined by $\varphi_{j}=\varphi$, with $\varphi=0$ for $\alpha<0, \varphi=\pi$ for $\alpha>0$, and any $\varphi$ for $\alpha=0$.

It can be shown that the function $E(\Phi)$, for $\alpha \neq 0$, may reach its minimum for alternating angles $\varphi_{j}=(-1)^{j} \varphi$, $0 \leqslant|\varphi|<\pi$. The collinear LECs, $\varphi=0$ or $\pi$, are nondegenerated, whereas solutions with other values of $\varphi$ are two-fold degenerated. The minimum condition yields a simple relation $\alpha=-4 \cos \varphi$ or $\varphi=\pi-\arccos (\alpha / 4)$. Of course, it can be satisfied for $|\alpha| \leqslant 4$ only. Outside this domain, the boundary values are retained, i.e. $\phi=0$ for $\alpha<-4$ and $\phi=\pi$ for $\alpha>4$ (see Fig. 4). The latter ones are the same as in the non-frustrated domain, i.e. for $J_{j j+1}=-1$. The corresponding energies are $-n\left(\alpha^{2} / 8+\right.$ $1)$ and $-n(|\alpha|-1) \mid$, for $|\alpha| \leqslant 4$ and $|\alpha| \geqslant 4$, respectively. 

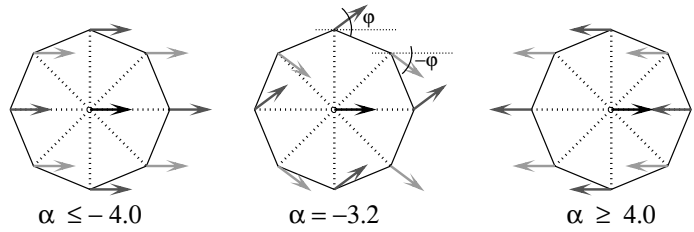

Fig. 4. The lowest energy configurations for a centered regular octagon; $J_{j+1}=1$ and $J_{0 j}=\alpha$.

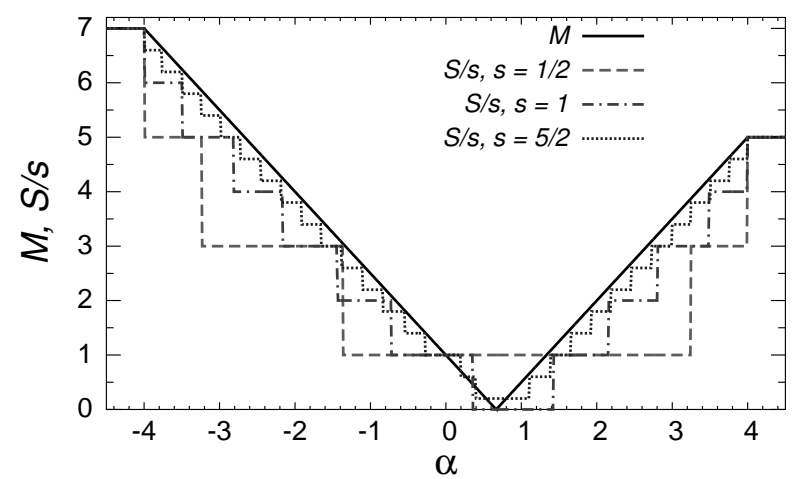

Fig. 5. An absolute value of the net magnetization $M=|\boldsymbol{M}|$ for a centered hexagon within the classical Heisenberg model compared with a relative total spin number $S / s$ in its quantum counterpart for $s=$ $1 / 2,1,5 / 2$; see also $[16,19,20]$.

The net magnetization for $|\alpha| \leqslant 4$ is equal to $(1-n \alpha / 4) \boldsymbol{s}_{0}$ and it is constant outside this domain with $M=(1-$ $\varepsilon n) s_{0}$, where $\varepsilon$ denotes the sign of $\alpha$. Hence, the nonmagnetic LEC is realized for $\alpha=4 / n$ (see Fig. 5).

\section{Summary}

It has been shown that in both systems studied in this paper there are domain(s) of the energy parameter $\alpha$, where the collinear (so non-degenerated) LECs are realized in geometrically frustrated systems. Moreover, these configurations are the same as these observed in the corresponding systems without frustration. Therefore, they can be considered as realizations of the third type frustration in classical systems $[10,14,16]$. Continuous changes of configurations start (and end, in the case of centered rings) at the well-determined critical value of $\alpha$, independent on system size for centered rings. It should to be stressed that these obtained for the second of models, $\left|\alpha_{\mathrm{c}}\right|=4$, are identical as those found in their quantum counterparts $[16,19,20]$. Basing on numerical results, it can be said that the first critical value for quantum rings with a defect bond tends to calculated here $\alpha_{\mathrm{c}}=1 /(n-1)$ (see Fig. 6 in Ref. [16]). Also other properties of quantum systems (e.g. the total spin number presented in Figs. 4 and 5) show good convergence to their classical values as $s \rightarrow \infty$.

\section{References}

[1] G. Toulouse, in: Spin Glass Theory, Beyond, Eds. M. Mézard, G. Parisi, M.A. Virasoro, World Sci., Singapore 2004, p. 99 [reprinted from Comm. Physique 2, 115 (1997)].

[2] J. Vannimenus, G. Toulouse, J. Phys. C: Solid State Phys. 10, L537 (1977).

[3] S. Kirkpatrick, Phys. Rev. B 16, 4630 (1977).

[4] H.T. Diep, H. Giacomini, in: Frustrated Spin Systems, Ed. H.T. Diep, World Sci., Singapore 2013, p. 1.

[5] L.S. Campana, A. Caramico D'Auria, M. D'Ambrosio, U. Esposito, L. De Cesare, G. Kamieniarz, Phys. Rev. B 30, 2769 (1984).

[6] P. Lacorre, J. Phys. C: Solid State Phys. 20, L775 (1987).

[7] O. Kahn, Chem. Phys. Lett. 265, 109 (1997).

[8] J. Schnack, Dalton Trans. 39, 4677 (2010).

[9] Y. Furukawa, K. Kiuchi, K. Kumagai, Y. Ajiro, Y. Narumi, M. Iwaki, K. Kindo, A. Bianchi, S. Carretta, P. Santini, F. Borsa, G.A. Timco, R.E.P. Winpenny, Phys. Rev. B 79, 134416 (2009).

[10] M.L. Baker, G.A. Timco, S. Piligkos, J.S. Mathiesone, H. Mutka, F. Tuna, P. Kozłowski, M. Antkowiak, T. Guidi, T. Gupta, H. Rath, R.J. Woolfson, G. Kamieniarz, R.G. Pritchard, H. Weihe, L. Cronin, G. Rajaraman, D. Collison, E.J.L. McInnes, R.E.P. Winpenny, Proc. Nat. Acad. Sci. 109, 19113 (2012).

[11] P. Kozłowski, M. Antkowiak, G. Kamieniarz, J. Nanopart. Res. 13, 6093 (2011).

[12] M. Antkowiak, P. Kozłowski, G. Kamieniarz, G.A. Timco, F. Tuna, R.E.P. Winpenny, Phys. Rev. B

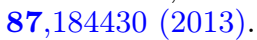

[13] P. Kozłowski, Phys. Rev. B 91, 174432 (2015).

[14] G. Kamieniarz, W. Florek, M. Antkowiak, Phys. Rev. B 92, 140411(R) (2015).

[15] E. Garlatti, S. Bordignon, S. Carretta, G. Allodi, G. Amoretti, R. De Renzi, A. Lascialfari, Y. Furukawa, G.A. Timco, R. Woolfson, R.E.P. Winpenny, P. Santini, Phys. Rev. B 93, 024424 (2016).

[16] W. Florek, M. Antkowiak, G. Kamieniarz, Phys. Rev. B 94, 224421 (2016).

[17] W. Florek, M. Antkowiak, G. Kamieniarz, K. Jaśniewicz-Pacer, Acta Phys. Pol. A $\mathbf{1 3 3}$, 411 (2018).

[18] H.-J. Schmidt, arXiv:1701.02489 (2017).

[19] A.M. Ako, O. Waldmann, V. Mereacre, F. Klöwer, I.J. Hewitt, Ch.E. Anson, H.U. Güdel, A.K. Powell, Inorg. Chem. 46, 756 (2007).

[20] J.W. Sharples, D. Collison, E.J.L. McInnes, J. Schnack, E. Palacios, M. Evangelisti, Nature Commun. 5, 5321 (2014). 